\title{
SOME ALGEBRAS OF ESSENTIALLY COMPACT DISTRIBUTIONS OF A REDUCTIVE P-ADIC GROUP
}

\author{
ALLEN MOY AND MARKO TADIĆ
}

\begin{abstract}
In this mainly expository paper, we review some convolution algebras for the category of smooth representations of $G$, and discuss their properties. Most important for us is the relation of these algebras with the Bernstein center algebra $\mathcal{Z}(G)$.
\end{abstract}

\section{In honor of Roger Howe as a sexagenarian}

\section{INTRODUCTION}

1.1. An indispensable tool in the representation theory of reductive Lie groups is to associate to an admissible representation $\pi$ of a connected reductive group $G$ a representation, also denoted as $\pi$, of the enveloping algebra $\mathfrak{U}(\operatorname{Lie}(G))$ of the Lie algebra $\operatorname{Lie}(G)$ of $G$. If the admissible representation $\pi$ is irreducible, then Schur's lemma states the center $\mathfrak{U}(\operatorname{Lie}(G))$ acts as scalar operators. The center $\mathcal{Z}(\mathfrak{U}(\operatorname{Lie}(G)))$ of $\mathfrak{U}(\operatorname{Lie}(G))$ can be viewed as the differential operators on the manifold $G$ which are left and right translation invariant, and this interpretation provides a concrete method to realize elements of the center. Furthermore, a fundamental result of Harish-Chandra determines the algebraic structure of the center $\mathcal{Z}(\mathfrak{U}(\operatorname{Lie}(G)))$.

An analogue of the center of the enveloping algebra for the representation theory of reductive p-adic groups has taken much longer to emerge, and is due to Bernstein (see [BD]). Certain aspects of the Bernstein center, in particular, explicit construction of elements in the center are still in a stage of development. Suppose $F$ is a non-archimedean local field of characteristic zero, i.e., a p-adic field, and $G=\mathrm{G}(F)$ the group of $F$-rational points of a connected reductive group $\mathrm{G}$. Let $\mathcal{C}_{c}^{\infty}(G)$ denote the vector space of locally constant compactly supported (complex valued) functions on $G$. We follow standard terminology and refer to a linear functional $D: \mathcal{C}_{c}^{\infty}(G) \longrightarrow \mathbb{C}$ as a distribution (see section

1991 Mathematics Subject Classification. Primary 22E50, 22E35.

The first author is partly supported by Research Grants Council grants HKUST6112/02P, and CERG \#602505. The second author is partly supported by Croatian Ministry of Science, Education and Sports grant \# 037-0372794-2804. 
2.1). Let $\mathcal{C}_{c}^{\infty}(G)^{*}$ denote the vector space of all distributions on $G$. Fix a choice of Haar measure on $G$. For $\theta \in C_{c}^{\infty}(G)$, let $D_{\theta}$ denote the distribution on $G$ which is integration of a function in $C_{c}^{\infty}(G)$ against $\theta$. If $f \in \mathcal{C}_{c}^{\infty}(G)$, then it is elementary (see section 2.3) the convolutions $f \star \theta$ and $\theta \star f$ can be expressed in terms of $D_{\theta}$ : Let $\breve{f}$ denote the function $x \rightarrow f\left(x^{-1}\right)$, and for $x \in G$, let $\lambda_{x}$, and $\rho_{x}$ denote left and right translations by $x^{-1}$, and $x$ respectively. Then

$$
\theta \star f=x \rightarrow D_{\theta}\left(\lambda_{x} \check{f}\right) \text { and } f \star \theta=x \rightarrow D_{\theta}\left(\rho_{x^{-1}} \check{f}\right) .
$$

These two formulae can then be extrapolated to provide a definition for the convolution of a distribution $D$ with any $f \in \mathcal{C}_{c}^{\infty}(G)$, i.e., $D \star f:=$ $x \rightarrow D\left(\lambda_{x} \check{f}\right)$ and $f \star D:=x \rightarrow D\left(\rho_{x^{-1}} \check{f}\right)$. In contrast to the case when the distribution arises from a $\theta \in \mathcal{C}_{c}^{\infty}(G)$, and the convolutions $\theta \star f$ and $f \star \theta$ belong to $\mathcal{C}_{c}^{\infty}(G)$, for an arbitrary distribution $D$, the functions $D \star f$ and $f \star D$ may not be compactly supported. A distribution $D$ of $C_{c}^{\infty}(G)$ is said to be essentially compact if the convolutions $D \star f$ and $f \star D$ are compactly supported functions for all $f \in C_{c}^{\infty}(G)$. In $[\mathrm{BD}]$, and [B], Bernstein-Deligne and Bernstein consider the space of essentially compact distributions which are $G$-invariant. The space of such distributions forms a convolution algebra known as the Bernstein center $\mathcal{Z}(G)$ of $G$.

1.2. In this mainly expository paper, we review some convolution algebras for the category of smooth representations of $G$, and discuss their properties. Most important for us is the relation of these algebras with the Bernstein center algebra $\mathcal{Z}(G)$.

For example in Bernstein's notes [B], he considers the Hecke algebra $\mathcal{H}(G)$ of compactly supported locally constant distributions, as well as the algebra $\mathcal{U}_{c}(G)$ of compactly supported distributions, and the endomorphism algebra $\operatorname{End}_{\mathbb{C}}\left(\mathcal{C}_{c}^{\infty}(G)\right.$ ) (see section 3.1). In [BD:§1.4], Bernstein, and Deligne consider the algebra

$$
\mathcal{H}(G)^{\wedge}:=\left\{D \in \mathcal{C}_{c}^{\infty}(G)^{*} \mid D \star f \in \mathcal{C}_{c}^{\infty}(G), \quad \forall f \in \mathcal{C}_{c}^{\infty}(G)\right\} .
$$

As part of the authors' investigations into the Bernstein center, we recently introduced in [MT2] the algebra

$\mathcal{U}(G):=\left\{D \in \mathcal{C}_{c}^{\infty}(G)^{*} \mid D \star f\right.$, and $\left.f \star D \in \mathcal{C}_{c}^{\infty}(G), \quad \forall f \in \mathcal{C}_{c}^{\infty}(G)\right\}$.

Obviously,

$$
\mathcal{H}(G) \subset \mathcal{U}_{c}(G) \subset \mathcal{U}(G) \subset \mathcal{H}(G)^{\wedge} .
$$

Furthermore, there is a natural monomorphism of $\mathcal{H}(G)^{\wedge}$ into the algebra $\operatorname{End}_{\mathbb{C}}\left(\mathcal{C}_{c}^{\infty}(G)\right)$, but the map is not an isomorphism (see section 3.2). The algebra $\mathcal{U}(G)$ obviously has a more symmetrical definition 
than $\mathcal{H}(G)^{\wedge}$, but both $\mathcal{U}(G)$, and $\mathcal{H}(G)^{\wedge}$ share many properties. Obviously, the center $\mathcal{Z}(\mathcal{U}(G))$ of $\mathcal{U}(G)$, and the center $\mathcal{Z}\left(\mathcal{H}(G)^{\wedge}\right)$ of $\mathcal{H}(G)^{\wedge}$, is the Bernstein center $\mathcal{Z}(G)$. We remark that $\mathcal{U}(G)$, like the enveloping algebra $\mathfrak{U}(\operatorname{Lie}(H))$ of a reductive Lie group $H$, has natural adjoint operation $*$. The algebra $\mathcal{H}(G)^{\wedge}$ does not have an adjoint operation.

We point out the similarity of the real and $p$-adic situations. The category of $\mathfrak{g}$-modules is equivalent to the category of $\mathfrak{U}(\mathfrak{g})$-modules. The center of this category, i.e., the algebra of all natural transformations of the identity functor, is isomorphic to the center of $\mathfrak{U}(\mathfrak{g})$. In particular, the center of $\mathfrak{g}$ is insufficient for describing the center of the category. A similar situation occurs in the $p$-adic case. The category $\operatorname{Alg}(G)$ of smooth representations of $G$ is equivalent to the category of non-degenerate modules over the Hecke algebra $\mathcal{H}(G)$ of $G$. But, neither the center of $G$, nor the center of $\mathcal{H}(G)$, is sufficient to describe the center of the category $\operatorname{Alg}(G)$. However, $\operatorname{Alg}(G)$ is also equivalent to the category of non-degenerate $\mathcal{U}(G)$-modules, and its center $\mathcal{Z}(\operatorname{Alg}(G))$ is isomorphic to the center $\mathcal{Z}(\mathcal{U}(G))=\mathcal{Z}(G)$ of $\mathcal{U}(G)$.

In [MT2], we mentioned some basic properties of $\mathcal{U}(G)$. Here, we provide proofs of those properties and establish additional properties of the convolution algebra $\mathcal{U}(G)$ and the closely related algebras mentioned above. We do this in section 3, after some preliminaries in section 2. Two highlights of section 3 are Theorem 3.4 o and Theorem 3.5e. The former states in particular for any smooth representation $(\pi, V)$ that $\pi\left(\mathcal{H}(G)^{\Upsilon}\right)$ equals $\operatorname{End}_{\mathbb{C}}(V)$. The latter states every $D \in \mathcal{H}(G)^{\wedge}$ is tempered.

In section 4 , we give some examples of explicit constructions of elements in the Bernstein center. It is rather hard but also rather important to describe explicitly distributions in the Bernstein center $\mathcal{Z}(G)$. These distributions are tempered and invariant. A big source of tempered invariant distributions are orbital integrals. These distributions are of principal interest in harmonic analysis on $G$, as well as in the modern theory of automorphic forms. Unfortunately, these distributions are rarely in the Bernstein center (see section 2.3). However, some natural linear combinations of the orbital integrals do belong the Bernstein center. In [MT2], the authors have constructed a large family of Bernstein center distributions in terms of orbital integrals. This is an interesting interplay between two types of very important distributions; namely, between orbital integral distributions, for which we have explicit formulas, but for which we do not have (in principle) explicit 
knowledge of their Fourier transforms, and Bernstein center distributions, for which (in principle) we know their Fourier transforms, but for which we have little explicit knowledge. We finish by formulating the main result of [MT2].

\section{The convolution algebras $\mathcal{H}(G)^{\wedge}$ and $\mathcal{U}(G)$}

2.1. Recall our already established notation from section 1: $F$ is a non-archimedean local field of characteristic zero, i.e., a p-adic field, $G=\mathrm{G}(F)$ the group of $F$-rational points of a connected reductive group $\mathrm{G}, \mathcal{C}_{c}^{\infty}(G)$ is the vector space of complex valued locally constant compactly supported functions on $G$, and $\mathcal{C}_{c}^{\infty}(G)^{*}$ is the space of complex linear functionals on $\mathcal{C}_{c}^{\infty}(G)$. The space $\mathcal{C}_{c}^{\infty}(G)$ can be viewed as having no topology. There is however a natural topology $\mathcal{T}$ on $\mathcal{C}_{c}^{\infty}(G)$, but all linear mappings are continuous with respect to $\mathcal{T}$. We briefly recall $\mathcal{T}$. Suppose $X$ is a non-empty open compact subset of $G$ and $J$ is an open compact subgroup of $G$. Define

$$
\begin{aligned}
\mathcal{V}_{X, J}:=\left\{f \in \mathcal{C}_{c}^{\infty}(G) \mid\right. & \text { (i) } \operatorname{supp}(f) \subset X, \\
& \text { (ii) } f \text { is } J \text {-bi-invariant } .
\end{aligned}
$$

The sets $\mathcal{V}_{X, J}$ are finite dimensional vector spaces. They have a natural topology on them (given, for example, by the standard supreme norm $\|f\|=\sup \{|f(x)| \mid x \in X\})$. A sequence of functions $f_{n}$ is said to converge to $f \in \mathcal{C}_{c}^{\infty}(G)$ precisely if there is a compact subset $X$ of $G$ and an open compact subgroup $J$ of $G$ so that all the $f_{n}$ 's and $f$ in are in $\mathcal{V}_{X, J}$, and we have convergence in that space. This defines the topology $\mathcal{T}$ on $\mathcal{C}_{c}^{\infty}(G)$. It follows, in particular, that any linear functional $D: \mathcal{C}_{c}^{\infty}(G) \rightarrow \mathbb{C}$ is continuous with respect to $\mathcal{T}$. We can alternatively define the topology $\mathcal{T}$ as the inductive topology (in the category of locally convex vector spaces) determined by requiring all embeddings $\mathcal{V}_{X, J} \hookrightarrow \mathcal{C}_{c}^{\infty}(G)$ be continuous.

Following standard usage, we refer to a linear functional $D \in \mathcal{C}_{c}^{\infty}(G)^{*}$ as a distribution.

2.2. Define the left and right translation action of $G$ on $\mathcal{C}_{c}^{\infty}(G)$ by

$$
\lambda_{g} f:=x \rightarrow f\left(g^{-1} x\right) \quad \text { and } \quad \rho_{g} f:=x \rightarrow f(x g)
$$

respectively. These two actions of $G$ on $\mathcal{C}_{c}^{\infty}(G)$ obviously commute with one another. A distribution $D$ is said to be $G$-invariant if $D(f)=$ $D\left(\lambda_{g} \rho_{g} f\right)$ for all $g \in G$. 
2.3. Suppose $\theta, f \in \mathcal{C}_{c}^{\infty}(G)$. Fix a choice of Haar measure on $G$. The convolution product $\theta \star f \in \mathcal{C}_{c}^{\infty}(G)$, which is a generalization of multiplication in the group algebra of a finite group, is defined as:

$$
\theta \star f:=x \longrightarrow \int_{G} \theta(g) f\left(g^{-1} x\right) d g .
$$

The distribution

$$
D_{\theta}(f):=\int_{G} \theta(g) f(g) d g
$$

satisfies

$$
\begin{aligned}
D_{\theta}(f) & =\int_{G} \theta(g) \check{f}\left(g^{-1}\right) d g, \text { where } \check{f}(g):=f\left(g^{-1}\right) \\
& =(\theta \star \check{f})(1) .
\end{aligned}
$$

We deduce

$$
(\theta \star f)=x \longrightarrow D_{\theta}\left(\lambda_{x}(\check{f})\right) .
$$

With $(2.3 \mathrm{~d})$ as a model, we define, for an arbitrary distribution $D$, and $f \in \mathcal{C}_{c}^{\infty}(G)$, the convolution $D \star f$ to be the function $G \rightarrow \mathbb{C}$ given by

$$
\begin{aligned}
D \star f: & =x \longrightarrow D\left(\lambda_{x}(\check{f})\right) \\
& =x \longrightarrow D\left(\text { function } t \rightarrow f\left(t^{-1} x\right)\right) .
\end{aligned}
$$

Similarly, we define

$$
\begin{aligned}
f \star D: & =x \longrightarrow D\left(\rho_{x^{-1}}(\check{f})\right) \\
& =x \longrightarrow D\left(\text { function } t \rightarrow f\left(x t^{-1}\right)\right) .
\end{aligned}
$$

If $D$ is $G$-invariant, then $D \star f=f \star D$. Both $D \star f$, and $f \star D$ are locally constant functions on $G$, but a-priori there is no reason they should be in $\mathcal{C}_{c}^{\infty}(G)$. An illuminating example of this is an orbital integral. Suppose $y \in G$. Let $\mathcal{O}:=\mathcal{O}(y)$ denote the conjugacy class of $y$. Then, $\mathcal{O}$ is a manifold isomorphic to the homogeneous space $G / C_{G}(y)$, where $C_{G}(y)$ is the centralizer of $y$ in $G$, and there is a $G$-invariant measure $d \mu_{\mathcal{O}}$ on $\mathcal{O}$, which is unique up to scalar. Then,

$$
\mu_{\mathcal{O}}(f):=\int_{\mathcal{O}} f(g) d \mu_{\mathcal{O}}(g)
$$

is a $G$-invariant distribution. If $1_{J}$ is the characteristic function of an open compact subgroup $J$, then $\lambda_{g} \check{1_{J}}$ is the characteristic function of $g J$, and

$$
\int_{\mathcal{O}} 1_{g J} d \mu_{\mathcal{O}}=\mu_{\mathcal{O}}(g J \cap \mathcal{O}) \text {. }
$$

In particular, the function $\mu_{\mathcal{O}} \star 1_{J}$ is compactly supported if and only if $\mathcal{O}$ is a compact orbit. An elementary argument then says for arbitrary 
$f \in \mathcal{C}_{c}^{\infty}(G)$, the convolution $\mu_{\mathcal{O}} \star f$ is compactly supported if and only if $\mathcal{O}$ is a compact orbit. An example of such compact orbits is the conjugacy class of a central element $z \in G$, for which the associated $G$-invariant distribution is the delta function $\delta_{z}$.

2.4. The Hecke algebra $\mathcal{H}(G)$ is the subspace of distributions $D \in$ $\mathcal{C}_{c}^{\infty}(G)^{*}$, satisfying

(i) $\operatorname{supp}(D)$ is compact, and

(ii) $D$ is locally constant, i.e., there exists a compact open subgroup $J_{D}$ of $G$ so that $D\left(\lambda_{g} f\right)=D(f)$, and $D\left(\rho_{g} f\right)=D(f) \forall g \in J_{D}$.

A choice of Haar measure on $G$ gives an identification of $\mathcal{H}(G)$ with $\mathcal{C}_{c}^{\infty}(G)$.

If $D$ is a compactly supported distribution, and $f \in \mathcal{C}_{c}^{\infty}(G)$, it is elementary both $D \star f$ and $f \star D$ are compactly supported functions. Furthermore, the function $D \star f$ (resp. $f \star D$ ) is right (resp. left) $J$ invariant for a sufficiently small open compact subgroup $J$.

Definition 2.4b A distribution $D$ is

(i) right essentially compact if $D \star f \in \mathcal{C}_{c}^{\infty}(G)$ for all $f \in \mathcal{C}_{c}^{\infty}(G)$,

(ii) left essentially compact if $f \star D \in \mathcal{C}_{c}^{\infty}(G)$ for all $f \in \mathcal{C}_{c}^{\infty}(G)$,

(iii) essentially compact if both $D \star f$ and $f \star D$ belong to $\mathcal{C}_{c}^{\infty}(G)$ for any $f \in \mathcal{C}_{c}^{\infty}(G)$.

We introduce three vector spaces of distributions. Following the Bernstein and Deligne [BD: $\S 1.4]$, set

$$
\mathcal{H}(G)^{\wedge}:=\left\{D \in \mathcal{C}_{c}^{\infty}(G)^{*} \mid D \star f \in \mathcal{C}_{c}^{\infty}(G) \quad \forall f \in \mathcal{C}_{c}^{\infty}(G)\right\} .
$$

Suppose $D_{1}, D_{2} \in \mathcal{H}(G)^{\wedge}$ and $f \in \mathcal{C}_{c}^{\infty}(G)$. To facilitate computations regarding compositions, let $C(f)$ denote the function $\check{f}$. Then, $D_{1} \star$ $\left(D_{2} \star f\right) \in \mathcal{C}_{c}^{\infty}(G)$, and we have the formula:

$$
\begin{aligned}
D_{2} \star\left(D_{1} \star f\right) & =x \rightarrow D_{2}\left(\lambda_{x}\left(C\left(D_{1} \star f\right)\right)\right) \\
& =x \rightarrow D_{2}\left(y \rightarrow\left(C\left(D_{1} \star f\right)\right)\left(x^{-1} y\right)\right) \\
& =x \rightarrow D_{2}\left(y \rightarrow\left(\left(D_{1} \star f\right)\right)\left(y^{-1} x\right)\right) \\
& =x \rightarrow D_{2}\left(y \rightarrow D_{1}\left(\lambda_{y^{-1} x}(C(f))\right)\right) \\
& =x \rightarrow D_{2}\left(y \rightarrow D_{1}\left(t \rightarrow C(f)\left(\left(y^{-1} x\right)^{-1} t\right)\right)\right) \\
& =x \rightarrow D_{2}\left(y \rightarrow D_{1}\left(t \rightarrow f\left(t^{-1} y^{-1} x\right)\right)\right) .
\end{aligned}
$$


If $D_{1}, D_{2} \in \mathcal{H}(G)^{\wedge}$, define their convolution product $D_{1} \star D_{2}$ as follows: For any $f \in \mathcal{C}_{c}^{\infty}(G)$,

$$
\begin{aligned}
\left(D_{2} \star D_{1}\right)(f): & =\left(D_{2} \star\left(D_{1} \star \check{f}\right)\right)(1) \\
& =D_{2}\left(y \rightarrow D_{1}(t \rightarrow f(y t))\right) .
\end{aligned}
$$

In particular, the function $x \rightarrow\left(D_{2} \star D_{1}\right)\left(\lambda_{x}(\check{f})\right)$ is precisely the function $D_{2} \star\left(D_{1} \star f\right)$. Thus, the convolution $\left(D_{2} \star D_{1}\right)$ is again in $\mathcal{H}(G)^{\wedge}$. To see that the convolution product is associative, we compute:

$$
\begin{aligned}
\left(D_{3} \star\left(D_{2} \star D_{1}\right)\right)(f) & =D_{3}\left(x \rightarrow\left(D_{2} \star D_{1}\right)(z \rightarrow f(x z))\right) \\
& =D_{3}\left(x \rightarrow D_{2}\left(y \rightarrow D_{1}(t \rightarrow f(x y t))\right),\right.
\end{aligned}
$$

and

$$
\begin{aligned}
\left(\left(D_{3} \star D_{2}\right) \star D_{1}\right)(f) & =\left(D_{3} \star D_{2}\right)\left(z \rightarrow D_{1}(t \rightarrow f(z t))\right) \\
& =D_{3}\left(x \rightarrow D_{2}\left(y \rightarrow D_{1}(t \rightarrow f(x y t))\right)\right) .
\end{aligned}
$$

The convolution product therefore makes $\mathcal{H}(G)$ into an algebra. We note that for any $g \in G$, the delta distribution $\delta_{g}$ at $g$ belongs to $\mathcal{H}(G)^{-}$, and the delta function $\delta_{1_{G}}$ at the identity $1_{G}$ is the identity element of $\mathcal{H}(G)^{\wedge}$. The Hecke algebra $\mathcal{H}(G)$ is a left ideal of $\mathcal{H}(G)^{\wedge}$, i.e., invariant under left multiplication by $\mathcal{H}(G)^{\wedge}$.

The algebra $\mathcal{H}(G)^{\wedge}$ (see [BD: $\left.\left.\S 1.4\right]\right)$ is a projective completion of the Hecke algebra $\mathcal{H}(G)$. As a (left-sided) analogue of the (right-sided) algebra $\mathcal{H}(G)^{\wedge}$, set

$$
\hat{\mathcal{H}}(G):=\left\{D \in \mathcal{C}_{c}^{\infty}(G)^{*} \mid f \star D \in \mathcal{C}_{c}^{\infty}(G) \quad \forall f \in \mathcal{C}_{c}^{\infty}(G)\right\} .
$$

As an analogue of $(2.4 \mathrm{~d})$, and $(2.4 \mathrm{e})$ we have

$$
\left(f \star D_{2}\right) \star D_{1}=x \rightarrow D_{2}\left(y \rightarrow D_{1}\left(t \rightarrow f\left(x t^{-1} y^{-1}\right)\right)\right)
$$

and

$$
\begin{aligned}
\left(D_{2} \star D_{1}\right)(f): & =\left(\left(C(f) \star D_{2}\right) \star D_{1}\right)(1) \\
& =D_{1}\left(t \rightarrow\left(C(f) \star D_{2}\right)\left(t^{-1}\right)\right) \\
& =D_{1}\left(t \rightarrow\left(D_{2}\left(y \rightarrow C(f)\left(t^{-1} y^{-1}\right)\right)\right)\right. \\
& =D_{1}\left(t \rightarrow\left(D_{2}(y \rightarrow f(y t))\right)\right) .
\end{aligned}
$$

In particular, $(2.4 \mathrm{i})$ defines an associative convolution product on the space $\hat{\mathcal{H}}(G)$. 
As a more symmetrical version of the two algebras $\mathcal{H}(G)^{\uparrow}$, and $\mathcal{H}(G)$, we set

$$
\begin{aligned}
\mathcal{U}(G): & =\mathcal{H}(G)^{\wedge} \cap{ }^{\wedge} \mathcal{H}(G) \\
& =\left\{D \in \mathcal{C}_{c}^{\infty}(G)^{*} \mid D \text { essentially compact }\right\} .
\end{aligned}
$$

We remark that for $D_{1}, D_{2} \in \mathcal{U}(G)$, formulae (2.4e) and (2.4i) provide two ways to define the convolution $D_{1} \star D_{2}$; namely as

$$
D_{1} \star_{r} D_{2}(f):=\left(D_{2} \star\left(D_{1} \star C(f)\right)\right)(1),
$$

and

$$
D_{1} \star_{l} D_{2}(f):=\left(\left(C(f) \star D_{2}\right) \star D_{1}\right)(1) .
$$

We show these two are the same. We first recall the identity $f_{1} \star f_{2}(1)=$ $f_{2} \star f_{1}(1)$ for any $f_{1}, f_{2} \in \mathcal{C}_{c}^{\infty}(G)$. Now, given $f \in \mathcal{C}_{c}^{\infty}(G)$, choose an sufficiently small open compact subgroup $J$ so that $e_{J} \star C(f)=$ $C(f)=C(f) \star e_{J}, e_{J} \star\left(D_{1} \star C(f)\right)=D_{1} \star C(f)=\left(D_{1} \star C(f)\right) \star e_{J}$, and $e_{J} \star\left(C(f) \star D_{2}\right)=C(f) \star D_{2}=\left(C(f) \star D_{2}\right) \star e_{J}$. Then,

$$
\begin{aligned}
D_{1} \star{ }_{r} D_{2}(f): & \left(D_{2} \star\left(D_{1} \star C(f)\right)\right)(1) \\
& =\left(D_{2} \star\left(e_{J} \star\left(D_{1} \star C(f)\right)\right)\right)(1) \\
& =\left(\left(D_{2} \star e_{J}\right) \star\left(e_{J} \star\left(D_{1} \star C(f)\right)\right)\right)(1) \\
& =\left(\left(D_{2} \star e_{J}\right) \star\left(e_{J} \star\left(D_{1} \star\left(e_{J} \star C(f)\right)\right)\right)\right)(1) \\
& =\left(\left(D_{2} \star e_{J}\right) \star\left(e_{J} \star D_{1}\right)\right) \star\left(e_{J} \star C(f)\right)(1) \\
& =\left(e_{J} \star C(f)\right) \star\left(\left(D_{2} \star e_{J}\right) \star\left(e_{J} \star D_{1}\right)\right)(1) \\
& =C(f) \star\left(\left(D_{2} \star e_{J}\right) \star\left(e_{J} \star D_{1}\right)\right)(1) \\
& =\left(C(f) \star\left(D_{2} \star e_{J}\right)\right) \star\left(e_{J} \star D_{1}\right)(1) \\
& =\left(\left(C(f) \star D_{2}\right) \star e_{J}\right) \star\left(e_{J} \star D_{1}\right)(1) \\
& =\left(\left(C(f) \star D_{2}\right) \star D_{1}\right)(1) \\
& =D_{1} \star_{l} D_{2}(f) .
\end{aligned}
$$

The Hecke algebra $\mathcal{H}(G)$ is a right ideal of ${ }^{\wedge} \mathcal{H}(G)$ and a two-sided ideal of $\mathcal{U}(G)$.

The center $\mathcal{Z}(\mathcal{U}(G))$ of $\mathcal{U}(G)$ is the subspace:

$$
\begin{aligned}
\mathcal{Z}(\mathcal{U}(G)) & =G \text {-invariant essentially compact distributions on } G \\
& =\mathcal{Z}(G), \text { the Bernstein center }
\end{aligned}
$$

This is also the center of $\mathcal{H}(G)^{\wedge}$ and ${ }^{\wedge} \mathcal{H}(G)$. 
2.5. Let $\mathcal{A}$ denote either $\mathcal{H}(G)^{\wedge}$, or $\mathcal{U}(G)$. For any $g \in G$, the delta function $\delta_{g}$ at $g$ belongs to $\mathcal{A}$. From this, we deduce that any (left) $\mathcal{A}$-module $V$ is a representation of the group $G$. Recall that if $J$ is an open compact subgroup of $G$, then the function

$$
e_{J}=\frac{1}{\operatorname{meas} J} 1_{J} \in \mathcal{C}_{c}^{\infty}(G)
$$

is an idempotent of $\mathcal{C}_{c}^{\infty}(G)$, i.e., $e_{J} \star e_{J}=e_{J}$. An $\mathcal{A}$-module $V$ is said to be non-degenerate if for any $v \in V$ there exists an open compact subgroup $J_{v}$ so that $e_{J_{v}} v=v$. Since $\delta_{g} \star e_{J_{v}}=e_{J_{v}}$ for all $g \in J_{v}$, it follows $\delta_{g} v=v$ for all $g \in J_{v}$. Thus, a non-degenerate representation of $\mathcal{A}$ is a smooth representation of $G$. Note that an $\mathcal{A}$-module $V$ is non-degenerate if and only if $V=\pi((\mathcal{H}(G))(V)$. The only if part is obvious. To see the if part, suppose $V=\pi((\mathcal{H}(G))(V)$, and $v \in V$. Write $v$ as $v=f w$, and take $L$ to be an open compact subgroup so that $f$ is $L$-left-invariant. Then, $\delta_{g} v=\delta_{g}(f w)=\left(\delta_{g} \star f\right) w=f w=v$.

Conversely, we now explain how a smooth representation $(\pi, V)$ leads to a non-degenerate representation of $\mathcal{A}$. Suppose $(\pi, V)$ is smooth, $v \in V$ and $D \in \mathcal{A}$. Choose a compact open subgroup $J$ so that $\pi(J) v=v$. The convolution product, $D \star e_{J}$, lies in the subspace $\mathcal{C}_{c}(G / J) \subset \mathcal{C}_{c}^{\infty}(G)$ of right $J$-invariant functions. Define

$$
\pi(D) v:=\pi\left(D \star e_{J}\right) v=\int_{G}\left(D \star e_{J}\right)(g) \pi(g)(v) d g .
$$

To see that $\pi(D)$ is well-defined is an elementary calculation. Suppose $L$ is an open compact subgroup of $J$. Then

$$
\begin{aligned}
e_{J} & =\frac{1}{\operatorname{meas}(J)} \sum_{L g \in L \backslash J} 1_{L} \star \delta_{g}, \text { so } \\
D \star e_{J} & =\frac{1}{\operatorname{meas}(J)} \sum_{L g \in L \backslash J} D \star 1_{L} \star \delta_{g} .
\end{aligned}
$$

Thus,

$$
\begin{aligned}
\pi\left(D \star e_{J}\right)(v) & =\frac{1}{\operatorname{meas}(J)} \sum_{L g \in L \backslash J} \pi\left(D \star 1_{L}\right) \pi(g)(v) \\
& =\frac{1}{[J: L] \cdot \operatorname{meas}(L)} \sum_{L g \in L \backslash J} \pi\left(D \star 1_{L}\right)(v) \\
& =\pi\left(D \star e_{L}\right)(v) .
\end{aligned}
$$

It follows $v \mapsto \pi(D) v$ is a well-defined action of the $\mathcal{A}$ on $V$. It is then elementary to show $\pi\left(D_{1}\right)\left(\pi\left(D_{2}\right) v\right)=\pi\left(D_{1} \star D_{2}\right) v$, i.e., $\pi: \mathcal{A} \longrightarrow$ 
$\operatorname{End}_{\mathbb{C}}(V)$ is a representation. Thus, a smooth representation of $G$ is precisely the an $\mathcal{A}$-module $V$ which is non-degenerate.

If $\left(\pi_{1}, V_{1}\right)$ and $\left(\pi_{2}, V_{2}\right)$ are two smooth representations, and $T$ : $V_{1} \longrightarrow V_{2}$ is a $G$-map, then

$$
T \pi_{1}(D)=\pi_{2}(D) T, \text { for any } D \in \mathcal{A} .
$$

To see this, suppose $v \in V_{1}$. Consider $v$, and $T(v)$. Choose an open compact subgroup $J$ which fixes both $v$ and $T(v)$, and consider $D \star e_{J}$. We have $\pi_{1}(D)(v)=\pi_{1}\left(D \star e_{J}\right)(v)$; hence, $T\left(\pi_{1}(D)\right)(v)=\left(T \circ \pi_{1}\right)(D \star$ $\left.e_{J}\right)(v)=\pi_{2}\left(D \star e_{J}\right)(T(v))=\pi_{2}(D)(T(v))$. When $D \in \mathcal{Z}(G)$, the operator $\pi(D)$ commutes with the action of $\pi$, i.e., $\pi(D) \in \operatorname{End}_{G}(V)$ so $\pi(D)$ is itself a $G$-morphism. In this way, to each $G$-invariant essentially compact distribution, there is a naturally attached endomorphism of each object in the category of smooth reprsentations, which commutes with the morphisms of the category.

2.6. The algebra $\mathcal{U}(G)$ is easily made into a $\star$-algebra as follows: For $f \in \mathcal{C}_{c}^{\infty}(G)$, define the adjoint $f^{\star} \in \mathcal{C}_{c}^{\infty}(G)$ to be $f^{*}(g):=\overline{f\left(g^{-1}\right)}$, and for $D \in \mathcal{U}(G)$, define the adjoint $D^{\star}$ to be the distribution $D^{\star}(f):=$ $\overline{D\left(f^{\star}\right)}$. In particular, the adjoint of the delta distribution $\delta_{g}$ is the delta distribution $\delta_{g^{-1}}$. It is not hard to see the $\star$-involution swaps $\mathcal{H}(G)^{\text {^ }}$, and ${ }^{\wedge} \mathcal{H}(G)$.

\section{Some properties of the Convolution algebras $\mathcal{H}(G)^{\wedge}$ AND $\mathcal{U}(G)$}

3.1. In this section we compare the algebras $\mathcal{H}(G)^{\wedge},{ }^{\wedge} \mathcal{H}(G)$, and $\mathcal{U}(G)$ to several related algebras. These other algebras are as follows.

Algebra of Distributions with compact support. This algebra of distributions is defined as

$$
\mathcal{U}_{c}(G):=\{D \in \mathcal{U}(G) \mid \operatorname{supp}(D) \text { is compact }\} .
$$

Clearly, $\mathcal{H}(G) \subset \mathcal{U}_{c}(G) \subset \mathcal{U}(G)$. We note that $\delta_{1_{G}} \in \mathcal{U}_{c}(G) \backslash \mathcal{H}(G)$.

Algebra of linear endomorphism of $\mathcal{C}_{c}^{\infty}(G)$. Set

$$
\operatorname{End}_{\mathbb{C}}\left(\mathcal{C}_{c}^{\infty}(G)\right):=\left\{\text { linear endomorphism of } \mathcal{C}_{c}^{\infty}(G)\right\} .
$$

Formula (2.2a) defines two commuting $G$-actions on $\operatorname{End}_{\mathbb{C}}\left(\mathcal{C}_{c}^{\infty}(G)\right)$; in particular, we can view $\operatorname{End}_{\mathbb{C}}\left(\mathcal{C}_{c}^{\infty}(G)\right)$ as a $G \times G$-module.

$$
(g, h) f:=x \rightarrow\left(\lambda_{g} \circ \rho_{h}\right)(f)(x)=f\left(g^{-1} x h\right)
$$


where $(g, h) \in G \times G, f \in \mathcal{C}_{c}^{\infty}(G)$. Set

$$
\begin{array}{r}
\operatorname{End}_{G \times G}\left(\mathcal{C}_{c}^{\infty}(G)\right):=\left\{T \in \operatorname{End}_{\mathbb{C}}\left(\mathcal{C}_{c}^{\infty}(G)\right) \mid T \circ \lambda_{g}=\lambda_{g} \circ T,\right. \\
\text { and } \left.T \circ \rho_{g}=\rho_{g} \circ T, \forall g \in G\right\} .
\end{array}
$$

3.2. Let $\mathcal{A}$ be either $\mathcal{H}(G)^{\wedge}$ or $\mathcal{U}(G)$. Given $D \in \mathcal{A}$, we obtain an element $T_{D} \in \operatorname{End}_{\mathbb{C}}\left(\mathcal{C}_{c}^{\infty}(G)\right)$ as follows:

$$
T_{D}(f):=D \star f \quad \text { which, by definition, is } x \rightarrow D\left(\lambda_{x}(\check{f})\right) \text {. }
$$

We have $T_{D_{1}}\left(T_{D_{2}}(f)\right)=D_{1} \star\left(D_{2} \star f\right)=\left(D_{1} \star D_{2}\right) \star f$, so the map

$$
D \rightarrow T_{D}
$$

is an algebra homomorphism of $\mathcal{A}$ into $\operatorname{End}_{\mathbb{C}}\left(\mathcal{C}_{c}^{\infty}(G)\right)$. Since we can recover the essentially compact linear functional $D$ from $T_{D}$ by the formula

$$
D(f)=T_{D}(\check{f})(1),
$$

the algebra homomorphism is an injection.

For arbitrary $T \in \operatorname{End}_{\mathbb{C}}\left(\mathcal{C}_{c}^{\infty}(G)\right)$, an extrapolation of formula (3.2c) defines a linear functional $D_{T}$ on $\mathcal{C}_{c}^{\infty}(G)$ as

$$
D_{T}(f):=T(\check{f})(1) \text {. }
$$

We apply formula (3.2a) to $D_{T}$ :

$$
T_{D_{T}}(f)=x \rightarrow D_{T}\left(\lambda_{x}(\check{f})\right)=T\left(\left(\lambda_{x}(\check{f})\right)^{\check{ }}\right)(1) .
$$

Since $\lambda_{x}(\check{f})=g \rightarrow \check{f}\left(x^{-1} g\right)=f\left(g^{-1} x\right)$, and so $\left(\lambda_{x}(\check{f})\right)^{\swarrow}=g \rightarrow f(g x)$, i.e., $\left(\lambda_{x}(\check{f})\right)^{\sim}=\rho_{x}(f)$. So,

$$
T_{D_{T}}(f)=x \rightarrow T\left(\rho_{x}(f)\right)(1) .
$$

It can be seen from this that, in general, the linear functional $D_{T}$ is not essentially compact. In particular, the algebra monomorphism (3.2b), considered on $\mathcal{H}(G)^{\wedge}$, is not onto. If $T$ satisfies $T \circ \rho_{y}=\rho_{y} \circ T$ for all $y \in G$, we conclude $T_{D_{T}}$ has the property that $T_{D_{T}} \star f$ equals $T(f)$ and so belongs to $\mathcal{C}_{c}^{\infty}(G)$ for all $f \in \mathcal{C}_{c}^{\infty}(G)$. This is one half the definition for the linear functional $D_{T}$ to be essentially compact. Similarly, if $T \circ \lambda_{y}=\lambda_{y} \circ T$ for all $y \in G$, then $f \star T_{D_{T}}$ equals $T(f)$. In particular, if $T \circ \rho_{y}=\rho_{y} \circ T$ and $T \circ \lambda_{y}=\lambda_{y} \circ T$ for all $y \in G$, then $f \star T_{D_{T}}=T(f)=T_{D_{T}} \star f$; so, the linear functional $D_{T}$ is both essentially compact and $G$-invariant, i.e., in the center of $\mathcal{A}$. Thus, the map (3.2b) is an isomorphism of $\mathcal{Z}(\mathcal{A})$ with $\operatorname{End}_{G \times G}\left(\mathcal{C}_{c}^{\infty}(G)\right)$, see [B].

At this point it is natural to recall the following theorem of Bernstein (see [BD:§1.9.1] as well as $[\mathrm{B}: \S 4.2]$ ). 
Proposition 3.2g. The center of the category $\operatorname{Alg}(G)$ of smooth representations of $G$ is isomorphic to $\mathcal{Z}(G)$.

Proof. An element $z$ of the center $\mathcal{Z}(\operatorname{Alg}(G))$, also called an endomorphism of the catgeory, is an assignment to each object, i.e., smooth representation $(\pi, V)$, a morphism $z(\pi): V \rightarrow V$ so that if $\left(\pi_{1}, V_{1}\right)$ and $\left(\pi_{2}, V_{2}\right)$ are two smooth representations and $\phi: V_{1} \rightarrow V_{2}$ is a morphism, then the following diagram commutes.

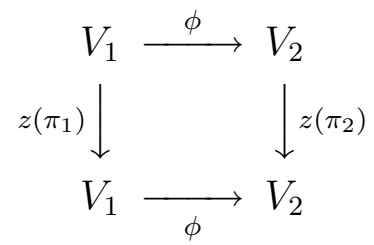

Suppose $D \in \mathcal{Z}(G)$. If $\left(\pi_{1}, V_{1}\right)$ and $\left(\pi_{2}, V_{2}\right)$ are smooth representations, and $\phi: V_{1} \longrightarrow V_{2}$ is a $G$-map, by $(2.5 \mathrm{e})$ we have $\pi_{2}(D) \circ \phi=\phi \circ \pi_{1}(D)$. Therefore, $\Gamma(D):=\pi \mapsto \pi(D)$ is an endomorphism of the category $\operatorname{Alg}(G)$. The map $D \rightarrow \Gamma(D)$, from $\mathcal{Z}(G)$ to $\mathcal{Z}(\operatorname{Alg}(G))$, is clearly a homomorphism of rings. We prove it is an isomorphism. We view $\mathcal{C}_{c}^{\infty}(G)$ as a smooth representation of $G$ via left translations $\lambda$.

Claim. The map $D \rightarrow \lambda(D)$ from $\mathcal{Z}(G)$ to $\{z(\lambda) \mid z \in \mathcal{Z}(\operatorname{Alg}(G))\}$ is an isomorphism.

For $D \in \mathcal{Z}(G)$, we have $\lambda(D)(f)=D \star f$; therefore, $D \rightarrow \lambda(D)$ is an injection. Conversely, suppose $T \in \operatorname{End}_{\lambda}\left(\mathcal{C}_{c}^{\infty}(G)\right)$ satisfies $T \circ \phi=\phi \circ T$ for any $G$-endomorphism of $\mathcal{C}_{c}^{\infty}(G)$. Any right translation $\rho_{g}$ is a $G$ endomorphism of $\mathcal{C}_{c}^{\infty}(G)$; therefore, $T \circ \rho_{g}=\rho_{g} \circ T$. Hence, we deduce $T \in \operatorname{End}_{G \times G}\left(\mathcal{C}_{c}^{\infty}(G)\right)$, and so there exists $D \in \mathcal{Z}(G)$ so that $T=T_{D}$. This proves the claim.

In particular, it follows the map $\Gamma$ is an injection. To prove $\Gamma$ is an isomorphism, it suffices to show any $z \in \mathcal{Z}(\operatorname{Alg}(G))$ is completely determined by $z(\lambda)$ (see also the remark in [BDK:§2.2]). To do this, choose $D \in \mathcal{Z}(G)$ so that $z(\lambda)=T_{D}$. Suppose $(\pi, V)$ is a smooth representation, $v \in V$, and $v$ is fixed by the open compact subgroup $J$. The map $\phi_{v}: \mathcal{C}_{c}^{\infty}(G) \rightarrow V$, defined as $\phi_{v}(f):=\pi(f) v$ is a $G$-map, and $\phi_{v}\left(e_{J}\right)=v$. Hence, $z(V) \circ \phi_{v}=\phi_{v} \circ z(\lambda)$; so, $z(V)(v)=z(V)\left(\phi_{v}\left(e_{J}\right)\right)=$ $\phi_{v}\left(z(\lambda)\left(e_{J}\right)\right)=\phi_{v}\left(D \star e_{J}\right)=\pi(D)\left(\phi_{v}\left(e_{J}\right)\right)=\pi(D)(v)$. We conclude $z(V)=\pi(D)$, and thus $\Gamma$ is an isomorphism as required.

3.3. Partition of the delta distribution $\delta_{1_{G}}$. Recall a sequence $\mathcal{J}=$ $\left\{J_{i}\right\}$ of decreasing open compact subgroups of $G$, i.e.,

$$
\mathcal{J}=\left\{J_{i}\right\} \text { with } J_{1} \supset J_{2} \supset \cdots \supset J_{i} \supset \cdots
$$


is cofinal among the neighborhoods of the identity, if given a neighborhood $\mathcal{V}$ of the identity, there exists a $J_{r}$ so that $\mathcal{V} \supset J_{r}$. For such a cofinal sequence $\mathcal{J}$, set

$$
\begin{aligned}
e_{i}=e_{\{\mathcal{J}, i\}} & :=\frac{1}{\operatorname{meas}\left(J_{i}\right)} 1_{J_{i}}, \\
\Delta_{i}=\Delta_{\{\mathcal{J}, i\}} & := \begin{cases}e_{1} & i=1 \\
e_{i}-e_{i-1} & i>1,\end{cases} \\
D_{\Delta_{i}}=D_{\Delta_{\{\mathcal{J}, i\}}} & :=\begin{array}{l}
\text { distribution associated } \\
\text { to } \Delta_{i} \text { as in }(2.3 \mathrm{~b}) .
\end{array}
\end{aligned}
$$

Note that

$$
\begin{aligned}
& \Delta_{i} \star \Delta_{j}=\delta_{i, j} \Delta_{i}, \\
& e_{i} \star \Delta_{j}=\Delta_{j} \star e_{i}=\left\{\begin{array}{cc}
\Delta_{j} & j \leq i, \\
0 & j>i .
\end{array}\right.
\end{aligned}
$$

Furthermore, if $(\pi, V)$ is a smooth representation of $G$, and $\operatorname{Im}\left(\pi\left(\Delta_{i}\right)\right)$ denotes the image subspace of the operator $\pi\left(\Delta_{i}\right)$, then $V$ decomposes as a direct sum

$$
V=\bigoplus_{i=1}^{\infty} \operatorname{Im}\left(\pi\left(\Delta_{i}\right)\right)
$$

and we have

$$
\pi\left(\Delta_{j}\right) v=\delta_{i, j} v \quad \text { for } \quad v \in \operatorname{Im}\left(\pi\left(\Delta_{i}\right)\right)
$$

Proposition 3.3g. Suppose $\mathcal{J}=\left\{J_{i}\right\}$ is a decreasing sequence of compact open subgroups of $G$ which is cofinal among the neighborhoods of the identity, and define $e_{i}, \Delta_{i}$, and $D_{\Delta_{i}}=D_{\Delta_{\{\mathcal{J}, i\}}}$ as in (3.3b). Then, for any $f \in \mathcal{C}_{c}^{\infty}(G)$, we have:

(i) For $i$ sufficiently large $\Delta_{i} \star f=0=f \star \Delta_{i}$. Equivalently, $D_{\Delta_{i}}(f)=0$ for $i$ sufficiently large.

(ii) $\sum_{i=1}^{\infty} \Delta_{i} \star f=f=\sum_{i=1}^{\infty} f \star \Delta_{i}$, and $\sum_{i=1}^{\infty} D_{\Delta_{i}}(f)=f(1)=$ $\delta_{1_{G}}(f)$. In particular, we have a decomposition of the delta distribution $\delta_{1_{G}}$ as

$$
\delta_{1_{G}}=\sum_{i=1}^{\infty} D_{\Delta_{i}} .
$$


Proof. Choose $N$ so that the function $f$ is $J_{N}$-bi-invariant. If $i \geq N$, then $J_{i} \subset J_{N}$, so $e_{i} \star f=f=f \star e_{i}$. This immediately implies (i) holds for $i>N$. The series of part (ii), when evaluated at $f \in \mathcal{C}_{c}^{\infty}(G)$, has only a finite number of non-zero terms; therefore, the equality is obvious.

3.4. The motivation for the next result is to take $D \in \mathcal{U}(G)$ and two partitions of the delta distribution at the identity, $\delta_{1_{G}}=\sum D_{\Delta_{\{\mathcal{J}, i\}}}$, and $\delta_{1_{G}}=\sum D_{\Delta_{\{\mathcal{K}, j\}}}$, and then justify the identity $D=\delta_{1_{G}} \star D \star \delta_{1_{G}}=$ $\sum_{i, j} D_{\Delta_{\{\mathcal{J}, i\}}} \star D \star D_{\Delta_{\{\mathcal{K}, j\}}}$.

Definition 3.4a. Let $C$ be an Abelian group, and $g_{i, j} \in C$ a two parameter family of elements of $C$. We say this family is locally finite if when we fix $i_{0}$, then the cardinality of $\left\{j \mid g_{i_{0}, j} \neq 0\right\}$ is finite, and when we fix $j_{0}$, then the cardinality of $\left\{i \mid g_{i, j_{0}} \neq 0\right\}$ is finite.

Proposition 3.4b. Suppose $\mathcal{J}=\left\{J_{i}\right\}$ and $\mathcal{K}=\left\{K_{j}\right\}$ are two decreasing sequences of compact open subgroups of $G$, with each sequence cofinal among the neighborhoods of the identity. Define $e_{\{\mathcal{J}, i\}}, \Delta_{\{\mathcal{J}, i\}}$, $D_{\Delta_{\{\mathcal{J}, i\}}}$ and $e_{\{\mathcal{K}, j\}}, \Delta_{\{\mathcal{K}, j\}}, D_{\Delta_{\{\mathcal{K}, j\}}}$ as in (3.3b). For any $D \in \mathcal{U}(G)$, set

$$
\Delta_{\{D,(\mathcal{J}, \mathcal{K}),(i, j)\}}:=\Delta_{\{\mathcal{J}, i\}} \star D \star \Delta_{\{\mathcal{K}, j\}} \in \mathcal{C}_{c}^{\infty}(G),
$$

and to $\Delta_{\{D,(\mathcal{J}, \mathcal{K}),(i, j)\}}$, let $D_{\{(\mathcal{J}, \mathcal{K}),(i, j)\}}$ be the associated distribution as in (2.3b). Then,

(i) Suppose $f \in \mathcal{C}_{c}^{\infty}(G)$. For $i+j$ sufficiently large, the two convolutions

$$
\Delta_{\{D,(\mathcal{J}, \mathcal{K}),(i, j)\}} \star f \text {, and } f \star \Delta_{\{D,(\mathcal{J}, \mathcal{K}),(i, j)\}}
$$

equal the zero function.

(ii) We have a decomposition of $D$ as

$$
D=\sum_{i, j} D_{\{(\mathcal{J}, \mathcal{K}),(i, j)\}} .
$$

Moreover, both of the two-parameter families $\Delta_{\{D,(\mathcal{J}, \mathcal{K}),(i, j)\}}$, as well as $D_{\{(\mathcal{J}, \mathcal{K}),(i, j)\}}$, are locally finite.

(iii) Suppose $g_{i, j} \in \mathcal{C}_{c}^{\infty}(G)$ for $i, j \geq 1$ is a locally finite collection of smooth functions. Set

$$
\Delta_{\left\{(\mathcal{J}, \mathcal{K}), g_{i, j}\right\}}:=\Delta_{\{\mathcal{J}, i\}} \star g_{i, j} \star \Delta_{\{\mathcal{K}, j\}},
$$


and let $D_{\Delta_{\left\{(\mathcal{J}, \mathcal{K}), g_{i, j}\right\}}}$ be the associated distribution as in (2.3b). Then, for any $f \in \mathcal{C}_{c}^{\infty}(G)$, for $i+j$ sufficiently large, we have $f \star \Delta_{\left\{(\mathcal{J}, \mathcal{K}), g_{i, j}\right\}}=0=\Delta_{\left\{(\mathcal{J}, \mathcal{K}), g_{i, j}\right\}} \star f$. In particular, holds $D_{\Delta_{\left\{(\mathcal{J}, \mathcal{K}), g_{i, j}\right\}}}(f)=0$, and

$$
D:=\sum_{i, j} D_{\Delta_{\left\{(\mathcal{J}, \mathcal{K}), g_{i, j}\right\}}}
$$

defines an essentially compact distribution.

(iv) Every essentially compact distribution is realizable in the form (3.4g).

(v) Suppose $g_{j} \in \mathcal{C}_{c}^{\infty}(G)$ for $j \geq 1$ is a collection of smooth functions. Set

$$
\Delta_{\left\{(\mathcal{K}, \mathcal{J}), g_{j}\right\}}:=\Delta_{\{\mathcal{J}, j\}} \star g_{j} \star \Delta_{\{\mathcal{K}, j\}},
$$

and let $D_{\Delta_{\left\{(\mathcal{K}, \mathcal{J}), g_{j}\right\}}}$ be the associated distribution as in (2.3b). Then, for any $f \in \mathcal{C}_{c}^{\infty}(G)$, for $j$ sufficiently large we have $f \star \Delta_{\left\{(\mathcal{K}, \mathcal{J}), g_{j}\right\}}=0=\Delta_{\left\{(\mathcal{K}, \mathcal{J}), g_{j}\right\}} \star f$. In particular, we have $D_{\Delta_{\left\{(\mathcal{K}, \mathcal{J}), g_{j}\right\}}}(f)=0$, and

$$
D:=\sum_{j} D_{\Delta_{\left\{(\mathcal{K}, \mathcal{J}), g_{j}\right\}}}
$$

is an essentially compact distribution.

Proof. (i) To prove (i), we have $\Delta_{\{D,(\mathcal{J}, \mathcal{K}),(i, j)\}} \star f=\Delta_{\{\mathcal{J}, i\}} \star D \star \Delta_{\{\mathcal{K}, j\}} \star f$. Choose $j_{0}$ so that $\Delta_{\{\mathcal{K}, j\}} \star f=0$ for $j \geq j_{0}$. For each $j$ in the range $1 \leq j<j_{0}$, choose $N_{j}$ so that if $i \geq N_{j}$, then $\Delta_{\{\mathcal{J}, i\}} \star D \star \Delta_{\{\mathcal{K}, j\}}=0$. Then, for $i+j \geq N_{r}:=\max \left\{N_{j} \mid 1 \leq j<j_{0}\right\}+j_{0}$, we have either $\Delta_{\{\mathcal{K}, j\}} \star f=0$ or $\Delta_{\{\mathcal{J}, i\}} \star D \star \Delta_{\{\mathcal{K}, j\}}=0$, hence $\Delta_{\{D,(\mathcal{J}, \mathcal{K}),(i, j)\}} \star f=0$. Similarly, there is a $N_{l}$ so that for $i+j \geq N_{l}$, we have $f \star \Delta_{\{D,(\mathcal{J}, \mathcal{K}),(i, j)\}}=$ 0 . Thus, if $i+j \geq \max \left(N_{r}, N_{l}\right)$ we have both $f \star \Delta_{\{D,(\mathcal{J}, \mathcal{K}),(i, j)\}}=0=$ $\Delta_{\{(\mathcal{J}, \mathcal{K}),(i, j)\}} \star f$, i.e., the assertion (i).

(ii) Formula (3.4d) is an immediate consequence of (i). Fix $i_{0}$. Then $\Delta_{\left\{\mathcal{J}, i_{0}\right\}} \star D \in C_{c}^{\infty}(G)$. Chose $j_{0}$ such that $\Delta_{\left\{\mathcal{J}, i_{0}\right\}} \star D$ is constant on left $K_{j_{0}}$-classes, i.e., on each $g K_{j_{0}}, g \in G$. Then, for $j>j_{0}$ we have $\Delta_{\left\{D,(\mathcal{J}, \mathcal{K}),\left(i_{0}, j\right)\right\}}=\left(\Delta_{\left\{\mathcal{J}, i_{0}\right\}} \star D\right) \star \Delta_{\{\mathcal{K}, j\}}=\left(\Delta_{\left\{\mathcal{J}, i_{0}\right\}} \star D\right) \star e_{K_{j_{0}}} \star \Delta_{\{\mathcal{K}, j\}}=0$ by (3.3d). In the same way one proves the second property for local finiteness. This implies the family $\Delta_{\{D,(\mathcal{J}, \mathcal{K}),(i, j)\}}$ is locally finite, and so the family $D_{\left\{(\mathcal{J}, \mathcal{K}),\left(i_{0}, j\right)\right\}}$ is locally finite.

(iii) Choose $i_{0}$ so that $f$ is constant on left $J_{i_{0}}$-classes. Then, for $i>i_{0}$, by (3.3d), we have $f \star \Delta_{\{\mathcal{J}, i\}}=0$, and so $f \star \Delta_{\{\mathcal{J}, i\}} \star g_{i, j} \star \Delta_{\{\mathcal{K}, j\}}=0$. Since $g_{i, j}$ is a locally finite family, we can find $j_{0}$ such that if $j>j_{0}$, 
then $g_{i, j}=0$ for all $i \leq i_{0}$. This imples that $f \star \Delta_{\{\mathcal{J}, i\}} \star g_{i, j} \star \Delta_{\{\mathcal{K}, j\}}=0$ for $i+j>i_{0}+j_{0}$. Similarly, we prove $\Delta_{\{\mathcal{J}, i\}} \star g_{i, j} \star \Delta_{\{\mathcal{K}, j\}} \star f=0$ for sufficienly large $i+j$.

Consider now $f \star D=f \star\left(\sum_{i, j} D_{\Delta_{\left\{(\mathcal{J}, \mathcal{K}), g_{i, j}\right\}}}\right)=\sum_{i, j} f \star D_{\Delta_{\left\{(\mathcal{J}, \mathcal{K}), g_{i, j}\right\}}}$. By the previous paragraph, this is a finite sum. Furthermore, $f \star$ $D_{\Delta_{\left\{(\mathcal{J}, \mathcal{K}), g_{i, j}\right\}}}$ are compactly supported smooth functions; therefore, $f \star$ $D \in \mathcal{C}_{c}^{\infty}(G)$. Similarly, $D \star f \in \mathcal{C}_{c}^{\infty}(G)$. This proves $D \in U(G)$.

(iv) For $D \in \mathcal{U}(G)$ take $g_{i, j}=\Delta_{\{\mathcal{J}, i\}} \star D \star \Delta_{\{\mathcal{K}, j\}}$, use that $\Delta_{\{\mathcal{J}, i\}}$ and $\Delta_{\{\mathcal{K}, j\}}$ are idempotents, and apply (ii).

(v) This assertion is a special case of (iii).

We now give a description of the algebra $\mathcal{H}(G)^{\wedge}$ analogous to Proposition $3.4 \mathrm{~b}$ for $\mathcal{U}(G)$,

Proposition 3.4j. Let $\mathcal{K}=\left\{K_{j}\right\}$ be a decreasing sequence of compact open subgroups of $G$, cofinal among the neighborhoods of the identity. Define $e_{\{\mathcal{K}, j\}}, \Delta_{\{\mathcal{K}, j\}}, D_{\Delta_{\{\mathcal{K}, j\}}}$ as in (3.3b). For $D \in \mathcal{H}(G)^{\wedge}$, set

$$
\Delta_{\{D, \mathcal{K}, j\}}:=D \star \Delta_{\{\mathcal{K}, j\}} \in \mathcal{C}_{c}^{\infty}(G),
$$

and to $\Delta_{\{D, \mathcal{K}, j\}}$, let $D_{\{D, \mathcal{K}, j\}}$ be the associated distribution as in (2.3b). Then:

(i) Suppose $f \in \mathcal{C}_{c}^{\infty}(G)$. For $j$ sufficiently large, the convolution $\Delta_{\{D, \mathcal{K}, j\}} \star f$ is the zero function.

(ii) We have a decomposition of $D$ as

$$
D=\sum_{j} D_{\{D, \mathcal{K}, j\}}
$$

(iii) Suppose $\left\{g_{j} \in \mathcal{C}_{c}^{\infty}(G) \mid j \geq 1\right\}$ is a sequence of smooth functions. Let $D_{g_{j} \star \Delta_{\{\mathcal{K}, j\}}}$ be in (2.3b). Then, for any $f \in \mathcal{C}_{c}^{\infty}(G)$, for sufficiently large $j,\left(g_{j} \star \Delta_{\{\mathcal{K}, j\}}\right) \star f=0$, and so $D_{g_{j} \star \Delta_{\{\mathcal{K}, j\}}}(f)=0$, and

$$
D:=\sum_{j} D_{g_{j} \star \Delta_{\{\mathcal{K}, j\}}}
$$

is in $\mathcal{H}(G)^{\wedge}$.

(iv) Every distribution in $\mathcal{H}(G)^{\wedge}$ is realizable in the form (3.4m). 
Proof. Observe that (i) follows from (i) of Proposition 3.3g. Further, (ii) follows from (ii) of the same proposition. The third claim follows from (i) of the same proposition. The last claim follows from the first two claims.

Set $S_{k}:=\sum_{j=1}^{k} D_{g_{j} \star \Delta_{\{\mathcal{K}, j\}}}$. We observe that

$$
S_{k+1} * e_{\{\mathcal{K}, k\}}=S_{k} .
$$

Therefore, in the above proposition we are working implicitely with elements of the projective limit.

Recall that if $V$ is an infinite dimensional vector space and $W$ a non-trivial vector space, then the space $\operatorname{Hom}_{\mathbb{C}}(V, W)$ has dimension at least the continuum.

Theorem 3.4o. Suppose $(\pi, V)$ is a smooth representation of a (connected) reductive p-adic group $G$. Write $\pi$ also for the associated nondegenerate representation of $\mathcal{H}(G)^{\frown}$ on $V$. Then, we have:

(i)

$$
\begin{aligned}
&\left\{\pi(D) \mid D \in \mathcal{U}(G) \text { and } \operatorname{dim}_{\mathbb{C}}(\right.\pi(D) V)<\infty\} \\
& \subset\{\pi(D) \mid D \in \mathcal{H}(G)\},
\end{aligned}
$$

Equality holds if $\pi$ is admissible.

(ii)

$$
\pi(\mathcal{H}(G)) \subset \pi\left(\mathcal{U}_{c}(G)\right) \subset \pi(\mathcal{U}(G)) \subset \pi\left(\mathcal{H}(G)^{\wedge}\right) .
$$

(iii) If $\pi$ is irreducible, then

$$
\operatorname{End}_{\mathbb{C}}(V)=\pi\left(\mathcal{H}(G)^{\wedge}\right) .
$$

(iv) If $\pi$ is irreducible infinite dimensional, then all inclusions in (3.4q) are strict.

(v) If $\pi$ is an irreducible finite dimensional representation, then all inclusions in (3.4q) are actually equalities.

Proof. (i) Suppose $D$ belongs to the left hand side of $(3.4 \mathrm{p})$. The finite dimensionality hypothesis means we can choose an open compact subgroup $J$ so that $\pi(D)(V)$ is contained in the $J$-invariants $V^{J}$. Obviously, $\pi(D)=\pi\left(e_{J}\right) \pi(D)=\pi\left(e_{J} \star D\right)$, and $e_{J} \star D$ is in Hecke algebra, since $D \in \mathcal{U}(G)$. This proves (i).

(ii) This assertion is obvious, since we know these inclusions for the corresponding algebras. 
(iii) Suppose $J$ is an open compact subgroup of $G$. Write $V^{J}$ for the finite dimensional subspace of $V$ fixed by $J$. For convenience, we fix a Haar measure on $G$, and therefore an identification of the subspace $\mathcal{C}_{c}(G / / J) \subset \mathcal{C}_{c}^{\infty}(G)$ of $J$-bi-invariant functions with the Hecke algebra $\mathcal{H}(G / / J)$ of compactly supported locally constant distributions which are $J$-bi-invariant. Let $\pi^{J}$ denote the representation of $\mathcal{H}(G / / J)$ on $V^{J}$. The irreducibility hypothesis on $V$ means $\operatorname{End}_{\mathbb{C}}\left(V^{J}\right)=\pi^{J}(\mathcal{H}(G / / J))$. In particular, if $V$ is finite dimensional, then $V=V^{J}$ for some $J$, and (3.4r) follows.

Suppose $V$ is infinite dimensional. Recall that $V$ must have countable dimension. Indeed, for any choice of a sequence $\mathcal{K}=\left\{K_{\ell}\right\}$ as in (3.3a), each of the subspaces $\operatorname{Im}\left(\pi\left(\Delta_{\ell}\right)\right)$ is finite dimensional (since an irreducible smooth representation is admissible), and so $V$ has countable dimension. Set $s_{0}:=0$. For $\ell>0$, set

$$
s_{\ell}:=\operatorname{dim}\left(\operatorname{Im}\left(\pi\left(\Delta_{1}+\cdots+\Delta_{\ell}\right)\right)\right),
$$

and select a basis $v_{s_{\ell-1}+1}, \ldots, v_{s_{\ell}}$ for $\operatorname{Im}\left(\pi\left(\Delta_{\ell}\right)\right)$. That (3.3e) holds means the sequence $\left\{v_{k}\right\}$ is a basis for $V$. To show (3.4r), it is enough to show for an arbitrary sequence of $V$-vectors $\left\{w_{k}\right\}$, the existence of a $D \in \mathcal{H}(G)^{\wedge}$ so that $w_{k}=\pi(D)\left(v_{k}\right)$ for all $k$. Take a sequence $\mathcal{J}=\left\{J_{\ell}\right\}$ as in (3.3a) so that $w_{1}, \ldots, w_{s_{\ell}}$ are fixed by $J_{\ell}$. We can then find $Q_{\ell} \in \mathcal{H}\left(J_{\ell} \backslash G / K_{\ell}\right)$, the subspace of compactly supported $J_{\ell^{-}}$leftinvariant and $K_{\ell}$-right-invariant distributions, so that $\pi\left(Q_{\ell}\right)\left(v_{i}\right)=w_{i}$ for $1 \leq i \leq s_{\ell}$. Let

$$
D=\sum_{j=1}^{\infty} D_{Q_{j} \star \Delta_{\{\mathcal{K}, j\}}} .
$$

Then, $D \in \mathcal{H}(G)^{\wedge}$ by (iii) of Proposition 3.4j.

Fix $k \geq 1$. Take $j \geq 1$ so that $s_{j-1}+1 \leq k \leq s_{j}$. Then

$$
\begin{aligned}
\pi(D) v_{k} & =\sum_{i=1}^{\infty} \pi\left(Q_{i}\right) \pi\left(\Delta_{\{\mathcal{K}, i\}}\right) v_{k} \\
& =\pi\left(Q_{j}\right) \pi\left(\Delta_{\{\mathcal{K}, j\}}\right) v_{k}=\pi\left(Q_{j}\right) v_{k}=w_{k} .
\end{aligned}
$$

This proves (3.4r) when $V$ is infinite dimensional.

(iv) Suppose $\pi$ is irreducible and infinite dimensional. We observe that $\pi(\mathcal{H}(G))$ consists of finite rank operators, while $\pi\left(\mathcal{U}_{c}(G)\right)$ contains some operators with infinite dimensional rank, and therefore, $\pi(\mathcal{H}(G)) \subsetneq \pi\left(\mathcal{U}_{c}(G)\right)$.

We now prove $\pi(\mathcal{U}(G)) \subsetneq \pi\left(\mathcal{H}(G)^{\wedge}\right)$. Recall that the space of finite rank operators in $\operatorname{End}_{\mathbb{C}}(V)$ has dimension the continuum. Therefore, since $\mathcal{H}(G)$ is countable dimensional, we can find a finite rank operator 
$A$ on $V$ which is not in $\pi(\mathcal{H}(G))$. By (i), $A$ is not in $\pi(\mathcal{U}(G))$, while (iii) imples $A \in \pi\left(\mathcal{H}(G)^{\wedge}\right)$. This proves $\pi(\mathcal{U}(G)) \subsetneq \pi\left(\mathcal{H}(G)^{\curvearrowright}\right)$.

Since $\pi\left(\mathcal{U}_{c}(G)\right) \subset \pi(\mathcal{U}(G))$, and $\operatorname{End}_{\mathbb{C}}(V)=\pi\left(\mathcal{H}(G)^{\wedge}\right)$, the above strict inclusion $\pi(\mathcal{U}(G)) \subsetneq \pi\left(\mathcal{H}(G)^{\wedge}\right)$ means

$$
\pi\left(\mathcal{U}_{c}(G)\right) \subsetneq \operatorname{End}_{\mathbb{C}}(V) .
$$

We now give a direct proof of this statement, which we will then modify to show $\left.\pi\left(\mathcal{U}_{c}(G)\right) \subsetneq \pi(\mathcal{U}(G))\right)$.

Suppose $D \in \mathcal{U}_{c}(G)$ is a compactly supported distribution. Take $X \subset G$ to be a compact subset containing $\operatorname{supp}(D)$. Suppose $v \in V$. Choose an open compact subgroup $J$ which fixes $v$. Write the product set $X J$ as a disjoint union

$$
X J=\bigsqcup_{i=1}^{M} g_{i} J .
$$

Clearly, the distribution $D \star e_{J}$ has support contained in $X J$, and is $J$-right-invariant. It follows

$$
\begin{aligned}
\pi(D)(v) & =\pi\left(D \star e_{J}\right)(v) \in V_{\{X, v\}} \\
: & =\operatorname{span}\left\{\pi\left(g_{1}\right)(v), \ldots, \pi\left(g_{M}\right)(v)\right\} .
\end{aligned}
$$

Therefore, we have proved for a fixed compact subset $X \subset G$, and $v \in V$, there exists a finite dimensional subspace $V_{\{X, v\}} \subset V$ so that if $D \in\left(C_{c}^{\infty}(G)\right)^{*}$ has support in $X$, then $\pi(D) v \in V_{\{X, v\}}$.

We now apply Cantor's diagonal argument. Take a basis $\left\{v_{i}\right\}$ of $V$, and write $G=\cup_{i=1}^{\infty} X_{i}$ as a union of increasing compact subsets $X_{i}$. For each $X_{i}$, and $v_{i}$ choose a finite dimensional space $V_{\left\{X_{i}, v_{i}\right\}}$ so that if $D \in \mathcal{U}_{c}(G)$ with $\operatorname{supp}(D) \subset X_{i}$, then $\pi(D)\left(v_{i}\right) \in V_{\left\{X_{i}, v_{i}\right\}}$. Choose $w_{i} \in V$ so that $w_{i} \notin V_{\left\{X_{i}, v_{i}\right\}}$. There exists a linear transformation $T$ of $V$ so that $T\left(v_{i}\right)=w_{i}$.

CLAIM. If $D$ is a compactly supported distribution, then $\pi(D) \neq T$.

We prove the claim by contradiction. Suppose $D \in \mathcal{U}_{c}(G)$ is such that $\pi(D)=T$. Take $i$, so that $\operatorname{supp}(D) \subset X_{i}$. Then, $\pi(D)\left(v_{i}\right) \in V_{\left\{X_{i}, v_{i}\right\}}$, but $w_{i}=T\left(v_{i}\right)=\pi(D)\left(v_{i}\right) \notin V_{\left\{X_{i}, v_{i}\right\}}$. This is a contradiction. So, the claim is proved, and $(3.4 \mathrm{v})$ follows immediately.

We now refine the above proof of $(3.4 \mathrm{v})$, to show that $\pi\left(\mathcal{U}_{c}(G)\right) \subsetneq$ $\pi(\mathcal{U}(G))$. Define a strictly increasing sequence of indexes $t_{1}<t_{2}<\ldots$ as follows: Let $s_{\ell}:=\operatorname{dim}\left(\operatorname{Im}\left(\pi\left(\Delta_{1}+\cdots+\Delta_{\ell}\right)\right)\right)$ be as in $(3.4 \mathrm{~s})$. Choose $t_{1} \geq 1$ so that $v_{s_{t_{1}}} \notin V_{\left\{X_{s_{1}}, v_{s_{1}}\right\}}$. Then, recursively choose $t_{i+1}>t_{i}$ so $v_{s_{t_{i+1}}} \notin V_{\left\{X_{s_{i+1}}, v_{s_{i+1}}\right\}}$. For each $i \geq 1$ choose $g_{i} \in \mathcal{H}(G)$ such that 
$\pi\left(g_{i}\right) v_{s_{i}}=v_{s_{t_{i}}}$. Form the distribution

$$
D:=\sum_{i=1}^{\infty} D_{\Delta_{\left\{\mathcal{K}, t_{i}\right\}} \star g_{i} \star \Delta_{\{\mathcal{K}, i\}}} .
$$

By (v) of Proposition 3.4b, this is an essentially compact distribution, i.e., $D \in \mathcal{U}(G)$. For all $i=1,2, \ldots$, we have

$$
\begin{aligned}
\pi(D) v_{s_{i}} & =\sum_{k=1}^{\infty} \pi\left(\Delta_{\left\{\mathcal{K}, t_{k}\right\}} \star g_{k} \star \Delta_{\{\mathcal{K}, k\}}\right) v_{s_{i}} \\
& =\pi\left(\Delta_{\left\{\mathcal{K}, t_{i}\right\}} \star g_{i} \star \Delta_{\{\mathcal{K}, i\}}\right) v_{s_{i}} \\
& =\pi\left(\Delta_{\left\{\mathcal{K}, t_{i}\right\}} \star g_{i}\right) v_{s_{i}}=\pi\left(\Delta_{\left\{\mathcal{K}, t_{i}\right\}}\right) v_{s_{t_{i}}}=v_{s_{t_{i}}} .
\end{aligned}
$$

Suppose $\pi(D)=\pi\left(D_{c}\right)$ for some $D_{c} \in \mathcal{U}_{c}(G)$. Choose $i \geq 1$ such that $\operatorname{supp}\left(D_{c}\right) \subset X_{s_{i}}$. Then $\pi\left(D_{c}\right) v_{s_{i}} \in V_{\left\{X_{s_{i}}, v_{s_{i}}\right\}}$ by the choice of $V_{\left\{X_{s_{i}}, v_{s_{i}}\right\}}$. But $\pi\left(D_{c}\right) v_{s_{i}}=\pi(D) v_{s_{i}}=v_{s_{t_{i}}} \notin V_{\left\{X_{s_{i}}, v_{s_{i}}\right\}}$ by the choice of $t_{i}$. This is a contradiction, and therefore $\left.\pi\left(\mathcal{U}_{c}(G)\right) \subsetneq \pi(\mathcal{U}(G))\right)$. The proof of (iv) is now complete.

(v) Since $\pi$ is irreducible and finite dimensional, we have $\pi(\mathcal{H}(G))=$ $\operatorname{End}_{\mathbb{C}}(V)$.

3.5. In this section we show any essentially compact distribution is tempered, i.e., extends to a continuous linear functional of the Schwartz space $\mathscr{C}(G)$ of $G$. We begin by briefly recalling its definition. More details and proofs can be found in [W].

Let $A_{\emptyset}$ be denote a maximal split $F$-torus in $G$, and $M_{\emptyset}$ its $F$ centralizer. Denote the maximal compact subgroup of $M_{\emptyset}$ by ${ }^{\circ} M_{\emptyset}$. Fix a minimal $F$-parabolic subgroup $P$ of $G$ containing $A_{\emptyset}$. Let $K$ be a special good maximal compact subgroup of $G$. The selection of $P$ determines the set of simple roots (with respect to $A_{\emptyset}$ ), which further defines a cone $M_{\emptyset}^{+}$in $A_{\emptyset}$. Then we have Cartan decomposition

$$
G=\bigsqcup_{m \in M_{\emptyset}^{+} /{ }^{\circ} M_{\emptyset}} K m K \quad \text { (disjoint decomposition). }
$$

Thus, we have a bijection $K \backslash G / K$ onto $M_{\emptyset}^{+} /{ }^{\circ} M_{\emptyset} \subset M_{\emptyset} /{ }^{\circ} M_{\emptyset}$. This bijection we denote by $\sigma$. The quotient $M_{\emptyset} /{ }^{\circ} M_{\emptyset}$ is a lattice, and we fix a norm || || on this lattice, which is invariant for the action of the Weyl group of $A_{\emptyset}$. Denote by $\delta_{P}$ the modular character of $P$. Extend $\delta_{P}$ to a $K$-invariant function on $G$ via the Iwasawa decomposition, i.e., by the formula $\delta_{P}(p k)=\delta_{P}(p)$ for $p \in P$ and $k \in K$. Set $\Xi$ to be the $K$-spherical function 


$$
\Xi(g)=\int_{K} \delta_{P}(k g)^{1 / 2} d k
$$

We recall that $\Xi$ is the matrix coefficient of the $K$-spherical vector in the unitary principal series induced representation from the trivial character of $M_{\emptyset}$.

Denote by $\mathcal{C}^{\infty}(G)$ the space of complex locally constant functions on $G$. For $r$ a positive integer, and $f \in \mathcal{C}^{\infty}(G)$, set

$$
v_{r}(f):=\sup \left\{\frac{|f(g)|(1+\|\sigma(g)\|)^{r}}{\Xi(g)} \mid g \in G\right\} .
$$

For a fixed open compact subgroup $J$ of $K$, set

$$
\begin{aligned}
\mathscr{C}(G, J):=\left\{f \in \mathcal{C}^{\infty}(G) \mid\right. & \text { (i) } f \text { is } J \text {-bi-invariant, } \\
& \text { (ii) for every } \left.r, \quad v_{r}(f)<\infty\right\} .
\end{aligned}
$$

The functions $v_{r}$ define semi-norms on $\mathscr{C}(G, J)$, and the collection of these semi-norms yields a topology on $\mathscr{C}(G, J)$ so that it is a Fréchet space. Furthermore, functions in $\mathscr{C}(G, J)$ are square integrable, and thus the convolution of two such functions can be defined by the usual formula. The convolution again belongs to $\mathscr{C}(G, J)$, and multiplication is continuous. In this way, $\mathscr{C}(G, J)$ is a Fréchet algebra.

The system $\mathscr{C}(G, J)$, as $J$ runs over the open subgroups of $K$, is an inductive system in the category of locally convex topological vector spaces, and the Schwartz space $\mathscr{C}(G)$ is the inductive limit of this family. The Schwartz space is a complete locally convex space. Since the spaces $\mathscr{C}(G, J)$ are Fréchet algebras, the mapping $\left(f_{1}, f_{2}\right) \mapsto f_{1} \star f_{2}$ is a continuous linear mapping $\mathscr{C}(G) \rightarrow \mathscr{C}(G)$ whenever we fix either $f_{1}$ or $f_{2}$.

Clearly, $\mathcal{C}_{c}^{\infty}(G) \subset \mathscr{C}(G)$. A distribution $D$ on $G$ is said to be tempered, if it extends to a continuous linear functional on $\mathscr{C}(G)$. Each compactly supported distribution is tempered. We shall see that this is a special case of a more general fact:

Theorem 3.5e. Any distribution in $\mathcal{H}(G)^{\wedge}$ is tempered. In particular, any essentially compact distribution, and therefore, any $D \in \mathcal{Z}(\mathcal{U}(G))$, is tempered.

Proof. Let $D \in \mathcal{H}(G)^{\wedge}$. Suppose $f$ is in the Schwartz space $\mathscr{C}(G)$. Then, there exists an open compact subgroup $J$ so that $f$ is $J$-biinvariant. Since $D \in \mathcal{H}(G)^{\wedge}$, we have $D \star e_{J} \in \mathcal{C}_{c}^{\infty}(G)$, and so the convolution $\left(D \star e_{J}\right) \star \check{f}$ is defined. Set

$$
D^{\#}(f):=\left(\left(D \star e_{J}\right) \star \check{f}\right)(1) .
$$


We observe that if $f$ has compact support, then $D^{\#}(f)=D(f)$. Furthermore, if $L$ is an open compact subgroup of $J$, by associativity of convolution, and the hypothesis $f$, hence $\check{f}$ is $J$-bi-invariant, we have

$$
\begin{aligned}
\left(D \star e_{L}\right) \star \check{f} & =\left(D \star e_{L}\right) \star\left(e_{J} \star \check{f}\right)=\left(\left(D \star e_{L}\right) \star e_{J}\right) \star \check{f} \\
& =\left(D \star\left(e_{L} \star e_{J}\right)\right) \star \check{f} \\
& =\left(D \star e_{J}\right) \star \check{f},
\end{aligned}
$$

and so

$$
\left(\left(D \star e_{L}\right) \star \check{f}\right)(1)=\left(\left(D \star e_{J}\right) \star \check{f}\right)(1) .
$$

In particular, we conclude $D^{\#}$ is a well-defined extension of the linear functional $D$ to elements $f \in \mathscr{C}(G)$. To prove $D^{\#}$ defines a continuous extension, it is enough to prove its restriction to the subspace $\mathscr{C}(G, J)$ of $J$-bi-invariant functions is continuous. The map $D^{\#}: \mathscr{C}(G, J) \longrightarrow \mathbb{C}$ is the composition of three continuous maps

$$
f \mapsto \check{f} \mapsto\left(D \star e_{J}\right) \star \check{f} \mapsto\left(\left(D \star e_{J}\right) \star \check{f}\right)(1)
$$

and therefore continuous.

We remark that by slight modification, this proof also applies to the algebra $\hat{\mathcal{H}}(G)$ too.

\section{Some EXPLicit $G$-INVARIANT ESSENTIALLY COMPACT DISTRIBUTIONS}

4.1. The results of the sections 2 and 3 establish the algebras $\mathcal{H}(G)^{\wedge}$ and $\mathcal{U}(G)$ as suitable p-adic analogues of the enveloping algebra of the Lie algebra of a connected reductive Lie group. The center of each is precisely the Bernstein center of $G$-invariant essentially compact distributions. In the notes $[\mathrm{B}]$, Bernstein raised the problem of explicit construction of $G$-invariant essentially compact distributions. In this section we give examples of such distributions, ending with recent results of the authors [MT2].

4.2. We begin with an example of Bernstein's from his notes [B].

4.2a. Bernstein's example. Suppose $G=\operatorname{SL}(\mathrm{n})(F), \psi: F \rightarrow \mathbb{C}$ a nontrivial additive character, and $\theta$ is the continuous $G$-invariant function $\theta(g):=\psi(\operatorname{trace}(g))$. Then, the $G$-invariant distribution

$$
D_{\theta}(f):=\int_{G} \theta(g) f(g) d g, f \in \mathcal{C}_{c}^{\infty}(G) .
$$

is essentially compact. 
Proof. We observe that it is enough to show $\theta \star 1_{J}$ is compactly supported for any open compact subgroup $J$. This is because given $f \in$ $\mathcal{C}_{c}^{\infty}(G)$, there exists an open compact $J$ such that $e_{J} \star f=f=f \star e_{J}$. So, if $D_{\theta} \star e_{J} \in \mathcal{C}_{c}^{\infty}(G)$, then $D_{\theta} \star f=D_{\theta} \star\left(e_{J} \star f\right)=\left(D_{\theta} \star e_{J}\right) \star f \in \mathcal{C}_{c}^{\infty}(G)$. As a second observation, we note that it is enough to restrict $J$ to be congruence subgroups $K_{m}$ of the maximal compact $K=\mathrm{SL}(\mathrm{n})\left(\mathcal{R}_{F}\right)$. Here, $\mathcal{R}_{F}$ is the ring of integers in $F$. So, suppose $J=K_{m}$. To show

$$
D_{\theta} \star 1_{J}:=g \mapsto \int_{J} \theta(g x) d x
$$

is compactly supported, we use the Cartan decomposition $G=K A^{+} K$ to write $g$ as $g=k_{1} d k_{2}$, where $k_{1}, k_{2} \in K$, and $d$ is a diagonal matrix with ascending powers of the uniformizing element $\varpi$ on the diagonal

$$
d=\operatorname{diag}\left(\varpi^{-a_{1}}, \varpi^{-a_{2}}, \ldots, \varpi^{-a_{n}}\right) \quad, \quad a_{1} \geq a_{2} \geq \cdots \geq a_{n} .
$$

Then,

$$
\begin{aligned}
\int_{J} \psi(\operatorname{tr}(g x)) d x & =\int_{J} \psi\left(\operatorname{tr}\left(k_{1} d k_{2} x\right)\right) d x \\
& =\int_{J} \psi\left(\operatorname{tr}\left(d k_{2} x k_{1}\right)\right) d x \\
& =\int_{J} \psi(\operatorname{tr}(d k x)) d x \quad, \quad k:=k_{2} k_{1} .
\end{aligned}
$$

In the last line, we have used the fact that $K$ normalizes the subgroup $J=K_{m}$. To see why the integral vanishes for $g$, i.e., $d$ outside a bounded set, we consider the case of $\mathrm{SL}(2)$. This case illustrates the basic idea. Let $\wp$ denote the prime ideal in $\mathcal{R}_{F}$. We have:

$$
d k x=\left[\begin{array}{cc}
\varpi^{-a} & 0 \\
0 & \varpi^{a}
\end{array}\right]\left[\begin{array}{cc}
k_{1,1} & k_{1,2} \\
k_{2,1} & k_{2,2}
\end{array}\right]\left[\begin{array}{cc}
1+x_{1,1} & x_{1,2} \\
x_{2,1} & 1+x_{2,2}
\end{array}\right], x_{i, j} \in \wp^{m} .
$$

So,

$$
\begin{aligned}
& \operatorname{tr}(d k x)=\varpi^{-a}\left(k_{1,1}\left(1+x_{1,1}\right)+k_{1,2} x_{2,1}\right)+ \\
& \varpi^{a}\left(k_{2,1}\left(x_{1,2}\right)+k_{2,2}\left(1+x_{2,2}\right)\right) .
\end{aligned}
$$

We have

$$
\begin{gathered}
\psi(\operatorname{tr}(d k x))=\psi\left(\varpi^{-a}\left(k_{1,1}\left(1+x_{1,1}\right)+k_{1,2} x_{2,1}\right)\right) \cdot \\
\psi\left(\varpi^{a}\left(k_{2,1}\left(x_{1,2}\right)+k_{2,2}\left(1+x_{2,2}\right)\right)\right) \\
=\psi\left(\varpi^{-a} k_{1,1}\right) \cdot \psi\left(\varpi^{-a}\left(k_{1,1} x_{1,1}+k_{1,2} x_{2,1}\right)\right) . \\
\psi\left(\varpi^{a}\left(k_{2,1}\left(x_{1,2}+k_{2,2} x_{2,2}\right)\right)\right) \cdot \psi\left(\varpi^{a} k_{2,2}\right) .
\end{gathered}
$$


If $g$ is sufficiently large, i.e., the integer $a$ is large positive, then will $\psi\left(\varpi^{a}\left(k_{2,1}\left(x_{1,2}+k_{2,2} x_{2,2}\right)\right)\right)$ and $\psi\left(\varpi^{a} k_{2,2}\right)$ be identically 1 for all elements $x_{1,2}, x_{2,2} \in \wp^{m}$. Thus, for $a$ sufficiently large positive, we have

$$
\psi(\operatorname{tr}(d k x))=\psi\left(\varpi^{-a} k_{1,1}\right) \cdot \psi\left(\varpi^{-a}\left(k_{1,1} x_{1,1}+k_{1,2} x_{2,1}\right)\right) .
$$

The important term is the 2 nd term. We coordinatize the group $J$ by elements $x_{1,1}, x_{1,2}, x_{2,1} \in \wp^{m}$. Then

$$
\begin{aligned}
& \int_{J} \psi(\operatorname{tr}(d k x)) d x= \\
& \quad \int_{\wp^{m} \times \wp^{m} \times \wp^{m}} \psi\left(\varpi^{-a} k_{1,1}\right) \cdot \psi\left(\varpi^{-a}\left(k_{1,1} x_{1,1}+k_{1,2} x_{2,1}\right)\right) d x_{1,1} d x_{2,1} d x_{1,2}= \\
& \quad \int_{\wp^{m}} \psi\left(\varpi^{-a} k_{1,1}\right)\left(\int_{\wp^{m} \times \wp^{m}} \psi\left(\varpi^{-a}\left(k_{1,1} x_{1,1}+k_{1,2} x_{2,1}\right)\right) d x_{1,1} d x_{2,1}\right) d x_{1,2} .
\end{aligned}
$$

For $a$ sufficiently large, since $k \in \mathrm{SL}(2)\left(\mathcal{R}_{F}\right)$, the inner integral over $\wp^{m} \times \wp^{m}$ is clearly zero. Therefore, the distribution $D_{\theta}$ is essentially compact.

4.3. It is very tempting to try to generalize the distribution $g \mapsto$ $\psi(\operatorname{trace}(g))$ as follows:

(1) For $x \in G=\operatorname{SL}(\mathrm{n})(F)$, let $c_{1}(x)$ denote the trace of $x$, and more generally $c_{k}(x)$ the coefficient of the $t^{n-k}$ in the characteristic polynomial $p_{x}(t)$ of $x$. Consider the class functions and distributions

$$
\begin{aligned}
\theta_{k}(x) & :=\psi\left(c_{k}(x)\right) \\
D_{k}(f)=D_{\theta_{k}}(f) & :=\int_{G} \theta_{k}(x) f(x) d x
\end{aligned}
$$

Which $D_{k}$ belong to the Bernstein center? The class function $g \rightarrow c_{k}(x)$ is in fact the character of an irreducible finite dimensional $F$-representation of SL(n). Take $V=F^{n}$ to be the standard defining representation of $G=\operatorname{SL}(\mathrm{n})(F)$. For $0 \leq k \leq n$, consider the exterior power $\Lambda^{k} V$ representation of $G$. Then,

(i) It is an irreducible miniscule representation.

(ii) The trace of $g \in G$ on $\Lambda^{k} V$ is $c_{k}(g)$.

If a distribution $D$ is essentially compact, then, it is obvious, the distribution $\check{D}: f \mapsto D(\check{f})$ is also essentially compact. For $g \in \operatorname{SL}(\mathrm{n})(F)$, we have $c_{k}\left(g^{-1}\right)=c_{n-k}(g)$. It follows $D_{k} \in \mathcal{Z}(G)$ 
if and only if $D_{n-k} \in \mathcal{Z}(G)$. In particular, since $D_{1} \in \mathcal{Z}(G)$, we have $D_{n-1} \in \mathcal{Z}(G)$.

(2) More generally, suppose $\rho: G \rightarrow \operatorname{GL}(\mathrm{m})(F)$ is an irreducible representation of $G$. Does the class function

$$
\theta_{\rho}(g):=\psi(\operatorname{trace}(\rho(g)))
$$

define a distribution in the Bernstein center?

The next example shows these two generalizations are false.

4.3c. SL(4)(F) and the coefficient $c_{2}$. The distribution $\Theta$ associated to the class function $g \mapsto \psi\left(c_{2}(g)\right)$ is not essentially compact.

Proof. Fix a positive integer $m$ so that $\wp^{m}$ lies in the kernel of $\psi$. Take $J=K_{m}$, the conguence subgroup of level $m$. For

$$
d k=\left[\begin{array}{cccc}
\varpi^{-t} & 0 & 0 & 0 \\
0 & 1 & 0 & 0 \\
0 & 0 & 1 & 0 \\
0 & 0 & 0 & \varpi^{t}
\end{array}\right]\left[\begin{array}{cccc}
0 & 0 & 0 & 1 \\
0 & 0 & 1 & 0 \\
0 & 1 & 0 & 0 \\
1 & 0 & 0 & 0
\end{array}\right], t>0
$$

we show $\Theta \star 1_{J}(d k) \neq 0$ for arbitrarily large $t$. For

$$
g=\left[\begin{array}{llll}
a & b & c & d \\
e & f & g & h \\
i & j & k & l \\
m & n & o & p
\end{array}\right]
$$

$c_{2}(g)$ is

$$
\begin{aligned}
c_{2}(g)=(b e+c i & +d m+g j+h n+l o) \\
& -(a f+a k+a p+f k+f p+k p) .
\end{aligned}
$$

Now, we have

$$
d k x=\left[\begin{array}{cccc}
\varpi^{-t} x_{4,1} & \varpi^{-t} x_{4,2} & \varpi^{-t} x_{4,3} & \varpi^{-t}\left(1+x_{4,4}\right) \\
x_{3,1} & x_{3,2} & 1+x_{3,3} & x_{3,4} \\
x_{2,1} & 1+x_{2,2} & x_{2,3} & x_{2,4} \\
\varpi^{t}\left(1+x_{1,1}\right) & \varpi^{t} x_{1,2} & \varpi^{t} x_{1,3} & \varpi^{t} x_{1,4}
\end{array}\right] .
$$

So,

$$
\begin{aligned}
& c_{2}(d k x)=\varpi^{-t}\left(x_{4,2} x_{3,1}+x_{4,3} x_{2,1}-x_{4,1} x_{3,2}-x_{4,1} x_{2,3}\right) \\
& \quad+\left(\left(1+x_{4,4}\right)\left(1+x_{1,1}\right)+\left(1+x_{3,3}\right)\left(1+x_{2,2}\right)-x_{3,2} x_{2,3}-x_{4,1} x_{1,4}\right) \\
& \quad+\varpi^{t}\left(x_{3,4} x_{1,2}+x_{2,4} x_{1,3}-x_{3,2} x_{1,4}-x_{2,3} x_{1,4}\right) .
\end{aligned}
$$


The assumption $\wp^{m} \subset \operatorname{Ker}(\psi)$ means

$$
\begin{aligned}
& \psi\left(c_{2}(d k x)\right)= \\
& \quad \psi\left(\varpi^{-t}\left(x_{4,2} x_{3,1}+x_{4,3} x_{2,1}-x_{4,1} x_{3,2}-x_{4,1} x_{2,3}\right)\right) \cdot \psi(1)^{2} .
\end{aligned}
$$

The variables $x_{4,4}, x_{4,3}, x_{4,2}, x_{4,1}, x_{3,2}, x_{3,1}, x_{2,3}, x_{2,1}$ run freely over $\wp^{m}$. The resulting integral is a Kloosterman sum, and it is non-zero for $t>>0$. Hence, $\Theta \star 1_{J}(d k) \neq 0$ for $t>>0$, so the distribution $\Theta$ is not essentially compact.

Remark 4.3j. The above proof and counterexamples can be adapted to the following situations.

(1) Suppose $G=\operatorname{Sp}(2 m)$, and $\rho: G \longrightarrow \operatorname{GL}(2 \mathrm{~m})(F)$ the natural defining representation. Then, the $G$-invariant distribution associated to the class function $g \mapsto \psi(\operatorname{trace}(\rho(g)))$ is essentially compact.

(2) Suppose $E / F$ is a quadratic extension of $F$ and $G=\operatorname{SU}(2,1)$, and $\rho: G \longrightarrow \mathrm{GL}(2 \mathrm{~m})(E)$ the natural defining representation. Then, the $G$-invariant distribution associated (using Haar measure) to the class function $g \mapsto \psi\left(\operatorname{trace}_{E / F}(\operatorname{trace}(\rho(g)))\right)$ is not essentially compact.

4.4. One plentiful, but mysterious source of elements in the Bernstein center is the set of irreducible supercuspidal representations.

4.4a. Supercuspidal characters. Suppose $G=\mathrm{G}(F)$ is a semisimple group. If $(\pi, V)$ is an irreducible supercuspidal representation of $G$, then the character $\theta_{\pi}$ of $\pi$ is an element of the Bernstein center.

Proof. We may assume $\pi$ is infinite dimensional. The hypothesis $G$ is semisimple means $\pi$ is unitary. Let $\langle$,$\rangle be a G$-invariant hermitian form on the space $V$ of $\pi$, and let $\left\{v_{i} i \in \mathbb{N}\right\}$ be an orthonormal basis. We have

$$
\theta_{\pi}(g)=\sum_{i}\left\langle v_{i}, \pi(g) v_{i}\right\rangle
$$

Suppose $J$ is an open compact subgroup of $G$. We have

$$
\begin{aligned}
\theta_{\pi} \star e_{J}(h) & =\frac{1}{\operatorname{meas}(J)} \int_{J} \theta_{\pi}(h x) d x \\
& =\frac{1}{\operatorname{meas}(J)} \int_{J} \sum_{i}\left\langle v_{i}, \pi(h x) v_{i}\right\rangle d x \\
& =\frac{1}{\operatorname{meas}(J)} \int_{J} \sum_{i}\left\langle\pi\left(h^{-1}\right) v_{i}, \pi(x) v_{i}\right\rangle d x
\end{aligned}
$$


So,

$$
\theta_{\pi} \star e_{J}(h)=\sum_{i}\left\langle\pi\left(h^{-1}\right) v_{i}, \pi\left(e_{J}\right) v_{i}\right\rangle .
$$

The operator $\pi\left(e_{J}\right)$ projects $V_{\pi}$ to the finite dimensional space of $J$-fixed vectors. We may choose the orthogonal basis so the $\operatorname{span}\left\{v_{1}, \ldots, v_{r}\right\}$ is $V_{\pi}^{J}$. Then

$$
\theta_{\pi} \star e_{J}(h)=\sum_{i=1}^{r}\left\langle v_{i}, \pi\left(h^{-1}\right) v_{i}\right\rangle .
$$

The assumption that $\pi$ is supercuspidal means each of the matrix coefficients

$$
h \mapsto\left\langle\pi\left(h^{-1}\right) v_{i}, v_{i}\right\rangle
$$

is supported on a compact set. In particular, their finite sum, i.e., $\theta_{\pi} \star e_{J}$ has compact support.

4.5. As mentioned in section 2 , if $\mathcal{O}$ is a conjugacy class in a connected reductive p-adic group, the orbital integral distribution $(2.3 \mathrm{~g})$ is essentially compact if and only if $\mathcal{O}$ is compact. The authors have discovered for non-compact classes in $\operatorname{SL}(2)(F)$ that certain linear combination of orbital integral are essentially compact (see [MT1]). These combinations can be predicted by the asymptotical behavior of the orbits at infinity. Furthermore, the authors have obtained a generalization of the SL $(2)(F)$ results to hyperbolic conjugacy classes in quasi-split groups. We finish by formulating the main result of [MT2].

We assume $G$ is the group of $F$-rational points of a connected reductive quasi-split $F$-group $G$. Let $A_{\emptyset}$ be a maximal split $F$-torus, $M_{\emptyset}=C_{G}\left(A_{\emptyset}\right)$, and $B=P_{\emptyset}$ a Borel $F$-subgroup containing $M_{\emptyset}$. Let $D: M_{\emptyset} \longrightarrow \mathbb{R}$ denote the Weyl denominator.

For $t \in M_{\emptyset}$, define the normalized orbital integral of the conjugacy class $\operatorname{Ad}(G)(t)$ in the usual way, i.e.,

$$
F_{f}^{M_{\emptyset}}(t)=D(t)^{1 / 2} \int_{G / M_{\emptyset}} f\left(h t h^{-1}\right) d h .
$$

Then, the main result of [MT2] is the following:

4.5d. Linear combination of orbital integrals. Let $\gamma_{0}, \gamma \in M_{\emptyset}$. Suppose that $\gamma_{0}(w \cdot \gamma)$ is regular for every $w \in W_{G}\left(A_{\emptyset}\right)$. It means that if $w^{\prime} \in W$, and $w^{\prime}\left(\gamma_{0} w(\gamma)\right)=\gamma_{0} w(\gamma)$, then $w^{\prime}=1$. Then, the distribution

$$
f \mapsto \sum_{w \in W_{G}\left(A_{\emptyset}\right)} \operatorname{sgn}(w) \quad F_{f}^{M_{\emptyset}}\left(\gamma_{0} w(\gamma)\right), \quad \forall f \in C_{c}^{\infty}(G)
$$

belongs to the Bernstein center. 


\section{ACKNOWLEDGMENT}

The authors thank Dan Barbasch, and Gordan Savin for helpful discussions in regards to the examples of section 4. The second author completed the last stages of this work while visiting the E. Schrödinger Institute in Vienna. He thanks the institute, and its director J. Schwermer for their hospitality.

\section{REFERENCES}

[B] Bernstein, J. (written by K. Rumelhart), Draft of: Representations of $p$ adic groups, preprint.

[BD] Bernstein, J.,rédigé par Deligne, P., Le "centre" de Bernstein, in book "Représentations des Groupes Réductifs sur un Corps Local" written by J.-N. Bernstein, P. Deligne, D. Kazhdan and M.-F. Vignéras, Hermann, Paris, 1984.

[BDK] Bernstein, J., Deligne, P. and Kazhdan, D., Trace Paley-Wiener theorem for reductive p-adic groups, J. Analyse Math 47 (1986), 180-192.

[MT1] Moy, A., Tadić, M., Conjugacy class asymptotics, orbital integrals, and the Bernstein center: the case of SL(2), Represent. Theory 9 (2005), 327-353.

[MT2] Moy, A., Tadić, M., A construction of elements in the Bernstein center for quasi-split groups, preprint (August 2006).

[W] Waldspurger, J.-L., La formule de Plancherel pour les groupes p-adiques, J. Inst. Math. Jussieu 2 (2003), 235-333.

Department of Mathematics, The Hong Kong University of Science and Technology, Clear Water Bay, Hong Kong, Email: amoy@ust.hk

Department of Mathematics, University of Zagreb, BijeničKa 30, 10000 Zagreb, Croatia, Email: tadic@math.hr 